Journal for

ImmunoTherapy of Cancer

\title{
Case of Merkel cell carcinoma in a patient with pre-existing ILD
}

\author{
Rohan Maniar (D) , ${ }^{1}$ Michaela Anderson, ${ }^{2}$ Bret Taback, ${ }^{3}$ Shaheer Khan, \\ Diana McDonnell, ${ }^{1}$ Anjali Saqi, ${ }^{4}$ George W Niedt, ${ }^{5}$ Richard Carvajal ${ }^{1}$
}

To cite: Maniar R, Anderson M, Taback B, et al. Case of Merkel cell carcinoma in a patient with pre-existing ILD. Journal for ImmunoTherapy of Cancer 2020;8:e01672. doi:10.1136/ jitc-2020-001672

Accepted 25 0ctober 2020

\section{ABSTRACT}

Merkel cell carcinoma (MCC) is a rare neuroendocrine tumor of the skin with high rates of local recurrence and distant metastases despite treatment with traditional cytotoxic chemotherapies. The recent advances in immunotherapy, including the use of immune checkpoint blockade (ICB) has revolutionized treatment for this disease and resulted in durable responses for some patients. However, many patients, due to underlying conditions, have been insufficiently evaluated for potential use of immunotherapy. Here we present a case of ICB treatment with Programmed cell death protein 1 (PD-1) inhibition in a patient with underlying interstitial lung disease (ILD) and a new diagnosis of MCC. Through a multidisciplinary approach, we were able to maintain close monitoring with serial clinical and radiographical followup. The patient achieved a complete response though unrelated medical issues resulting in a treatment hold. At the last follow-up, the patient continued to experience a durable response without evidence of recurrence. This case describes the use of pembrolizumab, a PD-1 inhibitor for the treatment of MCC in a patient with underlying ILD. The use of active surveillance with a multidisciplinary approach resulted in successful treatment of MCC without exacerbation of the underlying ILD. commercial re-use. See rights and permissions. Published by BMJ.

${ }^{1}$ Department of Medicine, Division of Hematology/

Oncology, Columbia University Irving Medical Center, New York, New York, USA

${ }^{2}$ Department of Medicine, Division of Pulmonology and Critical Care, Columbia University Irving Medical Center, New York, New York, USA

${ }^{3}$ Department of Surgery, Division of Breast Surgery, Columbia University Irving Medical Center, New York, New York, USA ${ }^{4}$ Department of Pathology and Cell Biology, Columbia University Irving Medical Center, New York, New York, USA

${ }^{5}$ Department of Dermatology, Division of Dermatopathology, Columbia University Irving Medical Center, New York, New York, USA

Correspondence to

Dr Rohan Maniar;

rm3729@cumc.columbia.edu

\section{BACKGROUND}

Merkel cell carcinoma (MCC) is a rare, aggressive neuroendocrine tumor of the skin that typically affects older patients and those with lighter skin tones. The incidence of MCC has steadily risen over the last 20 years with an incidence rate that has increased from 0.5 cases per 100,000 persons per year to 0.7 cases per 100,000 persons per year between 2000 and $2013 .^{1}$ The disease is characterized by high rates of local recurrence and lymph node metastases. Eighty percent of cases are associated with infection with the Merkel cell polyoma virus $(\mathrm{MCPyV})$, resulting in malignant transformation, while the remaining cases are commonly due to ultraviolet radiation-associated DNA damage. ${ }^{2}$

Multiple chemotherapy regimens have been used in MCC despite limited data. Treatment regimens generally reflect those seen in small-cell carcinomas, given the similarity in cell morphology, and include platinum-based agents with etoposide, anthracyclines or taxanes. Overall response rates are generally between $40 \%$ and $60 \%$ with diminishing response rates after multiple lines of therapy. Duration of response is also short-lived with median progression-free survival of 94 days and median overall survival of 9.4 months. ${ }^{3}$ The emergence of immune checkpoint blockade (ICB) has led to similar overall response rates with more durable responses. Pembrolizumab, a programmed cell death protein 1 (PD-1) inhibitor, has an objective overall response rate of $56 \%$ with response duration ranging from 2.2 to 9.7 months in both MCPyV-positive and negative tumors. ${ }^{4}$ Similarly, avelumab, a programmed death ligand 1 (PD-L1) inhibitor, had an overall response rate of up to $62 \%$ and a preliminary median progression free survival (PFS) of 9.1 months in treatment-naive, metastatic MCC. ${ }^{5}$

Clinical trials with PD-1 inhibitor monotherapy have been associated with immunemediated pneumonitis in approximately $3 \%$ of patients, though adverse event rates vary significantly based on tumor type with rates as high as $5.8 \%$ and $4.8 \%$ for non-small-cell lung cancer (NSCLC) and renal cell carcinoma, respectively. ${ }^{6}$ Pneumonitis rates for patients with MCC treated with PD-1 or PD-L1 ranged from $0 \%$ to $6 \%$, though samples sizes were limited, given the rarity of the disease. ${ }^{57}$

\section{CASE REPORT}

A 71-year-old man with fibrotic interstitial lung disease (ILD) began experiencing several months of progressively enlarging skin nodules predominantly on the right ankle and foot around May 2019. On his initial evaluation in August 2019, he was noted to have a $3 \mathrm{~cm}$ erythematous nodule on the lateral right foot and $<1 \mathrm{~cm}$ nodules on the right posterior ankle with associated swelling. He had no appreciable inguinal lymphadenopathy.

With regard to his pulmonary history, the patient developed a persistent cough in June of 2017 that failed to respond to antibiotics. 


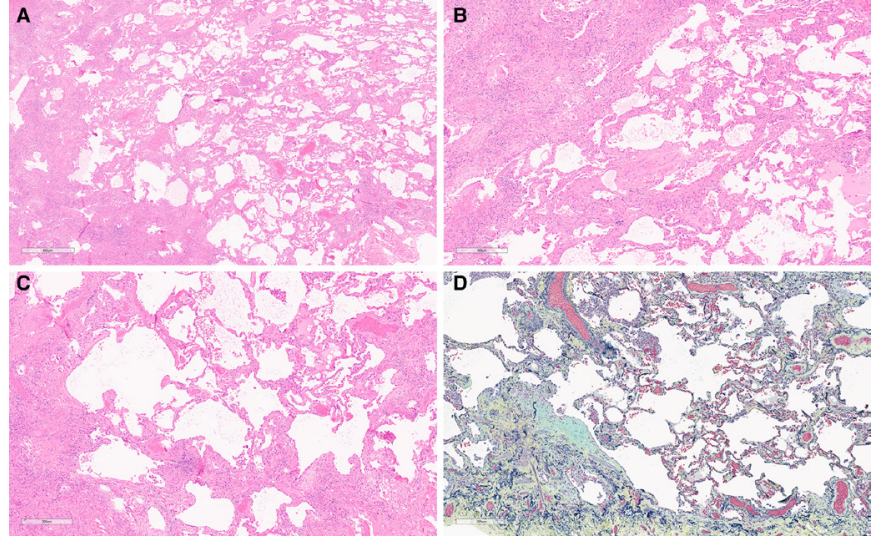

Figure 1 Right upper lobe wedge resection, July 2017. Usual interstitial pneumonia histology. (A-C) H\&E stain (800, 300 and $300 \mu \mathrm{m})$. Fibrosing and minimally cellular interstitial pneumonitis. (D) Movat stain $(300 \mu \mathrm{m})$ highlights the fibrosis (yellow) and fibroblastic foci (green).

A chest CT revealed basilar-predominant peripheral reticular changes with traction bronchiectasis but no honeycombing, suggestive of a usual interstitial pneumonia (UIP). Serological work-up was notable for a positive antinuclear antibody (ANA) (1:1280) with a nucleolar pattern (negative rheumatoid factor (RF), cyclic citrullinated peptie (CCP), anti-Jo-1, anti-Scl 70 antibody, anti-Sm antibody, anti-U1RNP antibody, anti-Ro/SSA antibody, and anti-La/SSB antibody), and surgical lung biopsy demonstrated fibrosing and minimally cellular interstitial pneumonitis with fibroblastic foci and airway-centered fibrosis most compatible with an UIP-type ILD (figure 1). He was evaluated by rheumatology who found no evidence of a connective tissue disease. A multidisciplinary discussion, including a review of radiographical, pathological and clinical findings, concluded this was most likely an idiopathic fibrotic non-specific interstitial pneumonia (NSIP). He initially declined therapy but, in 2018, developed progressively worsening dyspnea requiring $2 \mathrm{~L}$ of supplemental oxygen with exertion and a decline in forced vital capacity (FVC). Mycophenolate therapy was recommended; however, initiation was deferred due to pending elective surgical procedures. He was in this state of health when he developed these new lower extremity nodules in August 2019.

The patient was evaluated by dermatology and underwent punch biopsy, which revealed atypical cells with stippled nuclei and scant cytoplasm in a cohesive nodular and trabecular aggregation with positive CK20 and PCK in a paranuclear dot pattern consistent with MCC (figure 2). F18 Fluorodeoxyglucose (FDG) positron emission tomography (PET)/CT demonstrated FDG-avid skin lesions on the distal right leg and right ankle (Standardized Uptake Value (SUV) max 7.3) without evidence of metastatic disease (figure 3 ).

The patient was reviewed in multidisciplinary discussions with a determination to pursue systemic therapy, given a multifocal disease that was deemed unresectable and poor wound healing associated with radiation

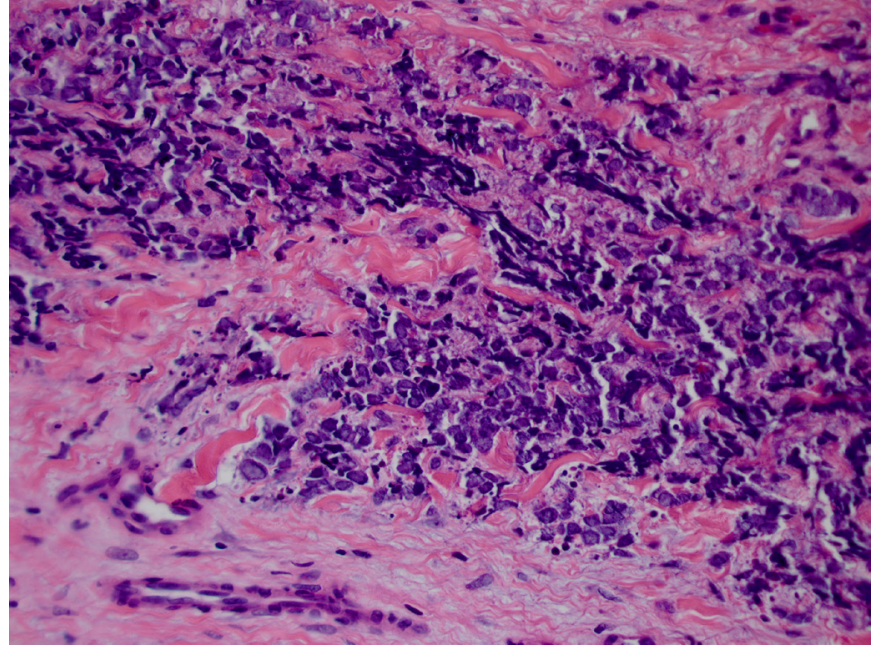

Figure 2 Right ankle punch biopsy, August 2019. Merkel cell carcinoma histology. H\&E stain $\times 40$.

therapy. Given the high rates of recurrence with standard chemotherapy and after long discussion regarding the potential risks of ICB in the setting of pre-existing ILD, the decision was made to pursue therapy with pembrolizumab. In discussion with his primary pulmonologist, it was opted to perform close pulmonary follow-up, including surveillance pulmonary function tests (PFTs) prior to each treatment and initiation of hydrofluoroalkane-beclomethasone dipropionate (HFA-BDP), based on particle size and high forward velocity seen with metered dose inhalers. Unfortunately, due to insurance

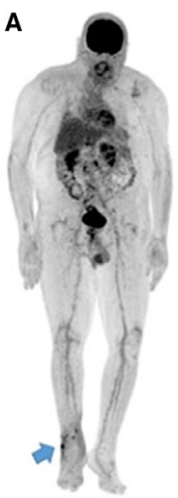

September 2019

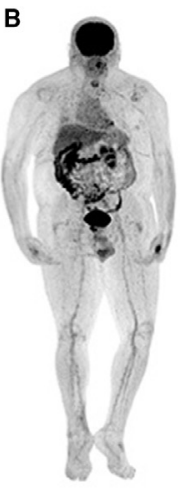

November 2019

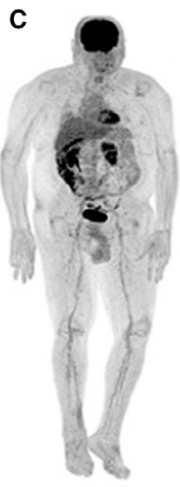

February 2020

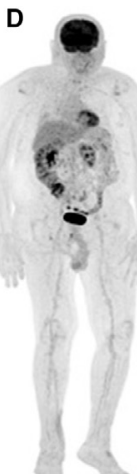

June 2019
Figure 3 Radiographical improvement in disease. (A) Baseline PET/CT from September 2019 showing FDGavid skin lesions in the distal right leg and ankle, with skin thickening and subcutaneous edema around the right ankle. (B) PET/CT from November 2019 on treatment with pembrolizumab showing overall improvement of disease of the right lower leg/ankle with resolution of previously seen raised anterolateral lesion, decreased size and metabolic activity of the anteromedial lesion and decreased thickening and subcutaneous edema. (C) PET/CT from February 2020 showing mild decreased FDG uptake associated with the right distal anteromedial leg. (D) PET/CT from June 2020 showing residual changes with edema around the right ankle; otherwise, no evidence of FDG-avid recurrent or metastatic disease. PET, positron emission tomography. 

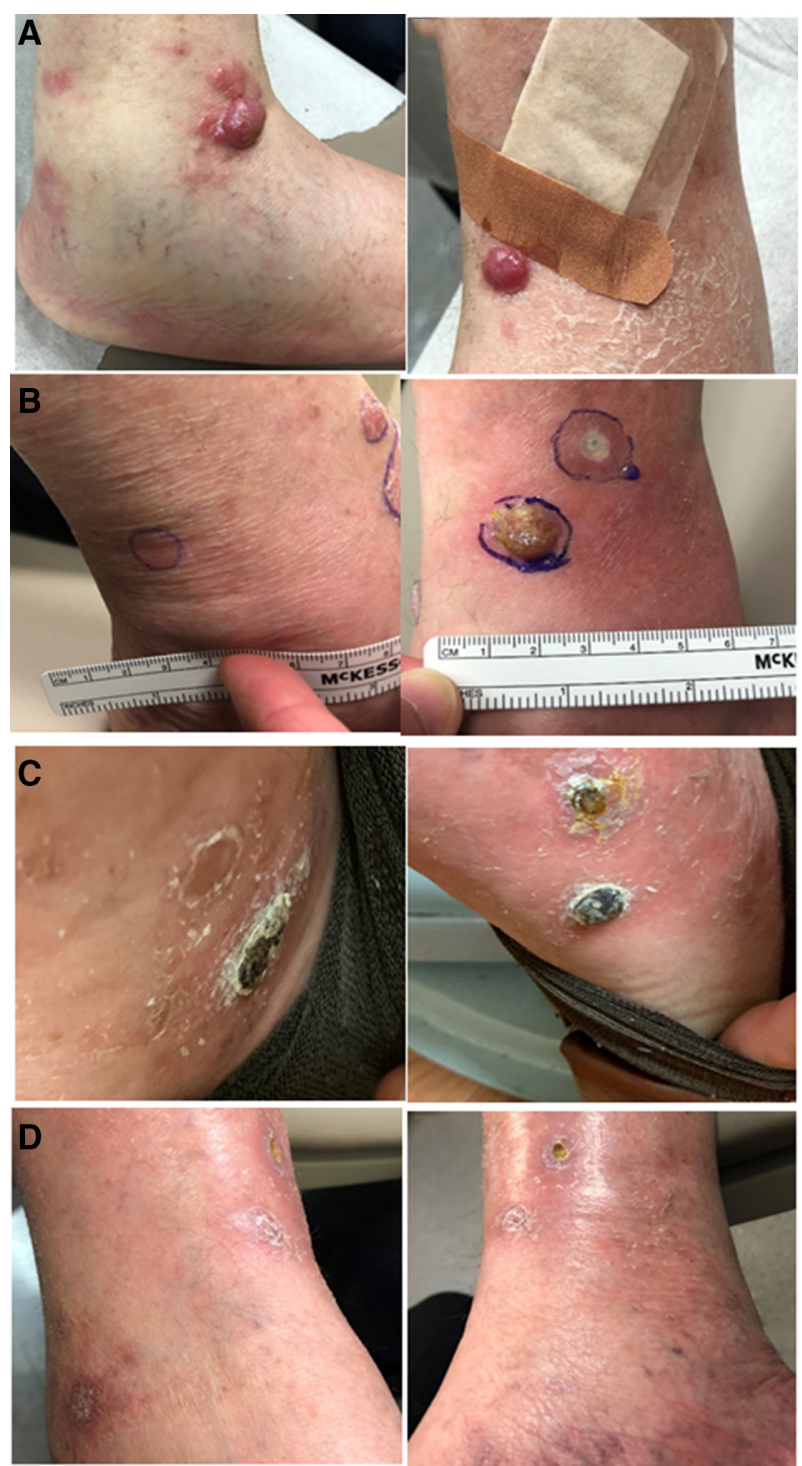

Figure 4 Clinical images of the right lower extremity. (A-D) Images showing lesions in the right lateral and left lateral aspect of the right lower extremity, respectively.

issues, that patient was maintained on fluticasone proprionate and salmeterol at a dosing of two times per day.

Initial PFTs prior to treatment revealed a diffusing capacity of lung carbon monoxide (DLCO) of $41 \%$ predicted $(12.57 \mathrm{~mL} / \mathrm{min} / \mathrm{mm} \mathrm{Hg})$. He started on pembrolizumab in September 2019 with good response after two cycles, including regression of the dominant lesion on the lateral aspect of the foot (figure 4). He underwent repeat PET/CT in November 2019, which demonstrated overall improvement of disease of the right lower leg and ankle with resolution of a raised anterolateral lesion, decreased size and metabolic activity of the anteromedial lesion and decreased skin thickening and subcutaneous edema without evidence of FDG-avid metastatic disease. The patient was maintained on serial PFT evaluations (figure 5) and clinical monitoring with no decrease in ambulation oxygen saturations.
Pulmonary Function Tests (PFTs)
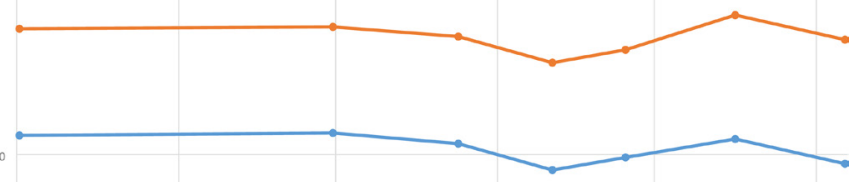

$8 / 2 / 2019$

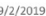

$11 / 2 / 2019$

12/2/2019

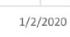

Figure 5 Graph highlighting DLCO on serial PFTs prior to and on-treatment with pembrolizumab. FVC, forced vital capacity; PFT, pulmonary function test.

In January 2020, the patient reported increased dyspnea with exertion and slightly decreased DLCO, for which treatment was held. A chest CT with intravenous contrast demonstrated stable fibrotic disease without evidence of an overlying acute parenchymal process or pulmonary embolism. His subsequent PET/CT showed persistent response. He ultimately resumed treatment with further delays in the setting of COVID-19. His treatment course was further complicated by a high-risk Non-ST elevation myocardial infarction on April 22, 2020, for which he underwent percutaneous coronary intervention with drug eluting stent placement. His last PET/CT in June 2020 demonstrated no evidence of FDG-avid recurrent or metastatic disease. Due to his complicated medical course, he is currently being observed expectantly off anticancer therapy.

\section{DISCUSSION}

This patient's high-risk disease with evolving lesions ultimately led to the decision to initiate systemic therapy. ICB offered the best opportunity for a meaningful treatment response with the potential for durable benefits. The possible benefits were weighed against the higher risk for immune therapy-related pneumonitis. A multidisciplinary discussion led to treatment with a PD-1 inhibitor, pembrolizumab.

For patients with MCC, ICB has emerged as a treatment option with durable responses. These promising new therapies, however, have not been evaluated in patients with pre-existing ILD and non-infectious pneumonitis. Several studies have attempted to evaluate the risk of ICB in high-risk patient populations, including those with preexisting ILD and UIP.

A retrospective study of 216 patients with NSCLC treated with nivolumab found pre-existing ILD in 26 patients, with the most common radiographical pattern of NSIP (14 patients) and UIP (12 patients). While median PFS rates were similar in the ILD and non-ILD groups (2.7 vs 2.9 months, $\mathrm{p}=0.919$ ), patients with pre-existing ILD experienced significant higher rates of treatment-related pneumonitis $(31 \%$ vs $12 \%, \mathrm{p}=0.014) .{ }^{8}$ Additionally, patients in 


\begin{tabular}{|c|c|c|c|c|}
\hline Case & Age (years) & Histology & Pre-existing ILD & Pneumonitis \\
\hline 1 & 72 & Squamous & UIP & AIP \\
\hline 3 & 72 & Adenocarcinoma & NSIP & COP \\
\hline 4 & 69 & Squamous & UIP & COP \\
\hline 6 & 74 & Adenocarcinoma & NSIP & COP \\
\hline 7 & 55 & Adenocarcinoma & NSIP & NSIP \\
\hline 8 & 65 & Squamous & NSIP & COP \\
\hline 1 & 71 & NSCLC-NOS & UIP & None \\
\hline 2 & 73 & NSCLC-NOS & Inconsistent UIP & None \\
\hline 6 & 64 & Squamous & UIP & None \\
\hline 1 & 67 & Squamous & Combined IPF & None \\
\hline
\end{tabular}

\section{All patients were males}

AIP, acute interstitial pneumonia; COP, cryptogenic organizing pneumonia; ICB, immune checkpoint blockade; ILD, interstitial lung disease; IPF, interstitial pulmonary fibrosis; NOS, not-otherwise specified; NSCLC, non-small-cell lung cancer; NSIP, non-specific interstitial pneumonia; UIP, usual interstitial pneumonia.

the ILD group experienced higher frequencies of severe reactions, defined as $\geq$ grade 3 , compared with the nonILD patients ( $19 \%$ vs $5 \%, \mathrm{p}=0.022)$ (table 1$)$.

In contrast, a prospective study in which six patients with NSCLC and mild interstitial pneumonitis were treated with nivolumab found no acute exacerbations in the underlying interstitial pneumonitis. ${ }^{9}$ A case report also highlights an experience of pre-existing ILD treated with nivolumab without associated pneumonitis. ${ }^{10}$

A review of the literature reveals scant data on the value of prophylactic regimens to help mitigate these risks or established surveillance practices for patients with pre-existing ILD. A randomized, double-blind, placebo-controlled, phase II study evaluated the use of prophylactic budesonide to prevent the onset of treatment induced colitis in patients with unresectable stage III or IV melanoma treated with ipilimumab. The study found no differences in grade $\geq 2$ diarrhea $(32.7 \%$ vs $35 \%$ ) between patients treated with ipilimumab/budesonide versus ipilimumab alone. ${ }^{11}$ Our decision to use prophylactic inhaled corticosteroids was based on a low probability for adverse effects, including limited ability to attenuate the activity of ICB based on absorption rates. We initially chose HFA-BDP based on the small particle size and propellant. Particles $1-5 \mu \mathrm{m}$ in diameter favor deposition in the small airways and alveoli with $>50 \%$ of the aerosolized medication being delivered to the alveoli. ${ }^{12}$ HFA-BDP contains the steroid in solution, rather than as suspension, which produces a mass median aerodynamic diameter of $1.1 \mu \mathrm{m} .{ }^{13}$
We conducted a surveillance plan that included pretreatment PFTs and frequent clinical evaluations to monitor symptoms. While there are no established guidelines for monitoring, an observational study of patients treated with ipilimumab for metastatic melanoma evaluated the use of surveillance PFTs. ${ }^{14}$ In this study, 80 patients underwent baseline and pretreatment PFTs during the course of their treatment. A reduction of FVC of $\geq 10 \%$ from baseline or a fall of $\geq 15 \%$ from baseline DLCO was deemed indicative of a possible pneumonitis, prompting high resolution CT of the chest. A significant decline in lung function occurred in 6/65 (9\%), 5/44 (11\%) and 9/38 (24\%) in patients after 3,6 and 9 weeks, respectively, with only 5 patients $(7 \%)$ reporting respiratory symptoms during this period. Nine patients underwent unplanned chest CT scan, which revealed progressive pulmonary metastases in five patients, normal radiographical findings in two patients and bilateral ground-glass opacities in one patient. The latter patient was ultimately diagnosed with ipilimumab-related pneumonitis.

As further studies expand the indications for ICB therapy, it will remain important to ensure that patients with comorbid conditions are not excluded from these beneficial treatment modalities. Prospective studies evaluating prophylactic regimens and establishing surveillance guidelines will be crucial to ensure that these patients gain the maximal benefit with mitigated risks.

Contributors RM and RC: writing of manuscript, figures and tables. MA, AS and GWN: editing of manuscript, figures. BT, SK and DMcD: editing of manuscript. 
Funding The authors have not declared a specific grant for this research from any funding agency in the public, commercial or not-for-profit sectors.

Competing interests None declared.

Patient consent for publication Obtained.

Provenance and peer review Not commissioned; externally peer reviewed.

Open access This is an open access article distributed in accordance with the Creative Commons Attribution Non Commercial (CC BY-NC 4.0) license, which permits others to distribute, remix, adapt, build upon this work non-commercially, and license their derivative works on different terms, provided the original work is properly cited, appropriate credit is given, any changes made indicated, and the use is non-commercial. See http://creativecommons.org/licenses/by-nc/4.0/.

\section{ORCID iD}

Rohan Maniar http://orcid.org/0000-0002-6089-2237

\section{REFERENCES}

1 Paulson KG, Park SY, Vandeven NA, et al. Merkel cell carcinoma: current US incidence and projected increases based on changing demographics. J Am Acad Dermatol 2018;78:457-63.

2 Tello TL, Coggshall K, Yom SS, et al. Merkel cell carcinoma: an update and review: current and future therapy. J Am Acad Dermatol 2018;78:445-54.

3 lyer JG, Blom A, Doumani R, et al. Response rates and durability of chemotherapy among 62 patients with metastatic Merkel cell carcinoma. Cancer Med 2016;5:2294-301.

4 Nghiem PT, Bhatia S, Lipson EJ, et al. PD-1 blockade with pembrolizumab in advanced Merkel-cell carcinoma. N Engl J Med 2016;374:2542-52.

5 D'Angelo SP, Russell J, Lebbé C, et al. Efficacy and safety of first-line Avelumab treatment in patients with stage IV metastatic Merkel cell carcinoma: a Preplanned interim analysis of a clinical trial. JAMA Oncol 2018;4:e180077.

6 Naidoo J, Wang X, Woo KM, et al. Pneumonitis in patients treated with Anti-Programmed Death-1/Programmed death ligand 1 therapy. J Clin Oncol 2017;35:709-17.

7 Nghiem P, Bhatia S, Lipson EJ, et al. Durable tumor regression and overall survival in patients with advanced Merkel cell carcinoma receiving pembrolizumab as first-line therapy. $J$ Clin Oncol 2019;37:693-702.

8 Kanai O, Kim YH, Demura Y, et al. Efficacy and safety of nivolumab in non-small cell lung cancer with preexisting interstitial lung disease. Thorac Cancer 2018;9:847-55.

9 Fujimoto $\mathrm{D}$, Morimoto $\mathrm{T}$, Ito $\mathrm{J}$, et al. A pilot trial of nivolumab treatment for advanced non-small cell lung cancer patients with mild idiopathic interstitial pneumonia. Lung Cancer 2017;111:1-5.

10 Khunger M, Velcheti V. A case of a patient with idiopathic pulmonary fibrosis with lung squamous cell carcinoma treated with nivolumab. $J$ Thorac Oncol 2017;12:e96-7.

11 Weber J, Thompson JA, Hamid O, et al. A randomized, double-blind, placebo-controlled, phase II study comparing the tolerability and efficacy of ipilimumab administered with or without prophylactic budesonide in patients with unresectable stage III or IV melanoma. Clin Cancer Res 2009;15:5591-8.

12 Labiris NR, Dolovich MB. Pulmonary drug delivery. Part I: physiological factors affecting therapeutic effectiveness of aerosolized medications. Br J Clin Pharmacol 2003;56:588-99.

13 Vanden Burgt JA, Busse WW, Martin RJ, et al. Efficacy and safety overview of a new inhaled corticosteroid, QVAR (hydrofluoroalkanebeclomethasone extrafine inhalation aerosol), in asthma. J Allergy Clin Immunol 2000;106:1209-26.

14 Franzen D, Schad K, Kowalski B, et al. Ipilimumab and early signs of pulmonary toxicity in patients with metastastic melanoma: a prospective observational study. Cancer Immunol Immunother 2018;67:127-34. 